\title{
Bleeding and management of coagulopathy
}

\author{
Roman M. Sniecinski, MD, and Jerrold H. Levy, MD, FAHA
}

\begin{abstract}
Bleeding after cardiac surgery remains a significant problem, increasing both length of stay and mortality, and is caused by multiple factors including dilutional changes, ongoing fibrinolysis, and platelet dysfunction. The evaluation of coagulopathy is problematic because of the long turnaround time of standard coagulation tests. Algorithms involving point of care testing, including thromboelastography and thromboelastometry, have been published; all have the potential to reduce transfusion requirements. Massive transfusion coagulopathy that occurs in trauma can also be seen in complex aortic surgery and other massive bleeding patients and should prompt consideration of a transfusion protocol involving fixed ratios of fresh frozen plasma, platelets, and red blood cells. Pharmacologic agents such as antifibrinolytics are commonly administered, but a multimodal approach to management is important. Recombinant and purified coagulation products are being studied and provide clinicians specific agents to treat targeted deficiencies. A general multi-modal approach is required and recommendations are made for the management of bleeding and coagulopathy in cardiac surgical patients. ( $\mathrm{J}$ Thorac Cardiovasc Surg 2011;142:662-7)
\end{abstract}

Approximately $5 \%$ to $7 \%$ of patients undergoing cardiac surgery lose more than $2 \mathrm{~L}$ of chest tube drainage during the first 24 hours postoperatively, and up to $5 \%$ require reexploration for bleeding, resulting in an increased length of stay and higher mortality. ${ }^{1-3}$ Multiple causes, including activation of the coagulation, fibrinolytic, and inflammatory pathways, dilutional changes, hypothermia, and other surgical factors, contribute to this problem. Bleeding may also be further exacerbated by perioperative use of various anticoagulants, including heparin, thrombin inhibitors, and platelet inhibitors. There is a complex equilibrium among red blood cells (RBCs), platelets, coagulation factors, natural inhibitors of coagulation, and the fibrinolytic system that is significantly altered in cardiac surgery. Therefore, simple replacement of blood volumes may not always be effective. This review will focus on current therapies to treat bleeding after cardiac surgery. An overview of the coagulation process, with potential sites of intervention, is presented in Figure 1.

\section{LABORATORY TESTING AND USE OF ALGORITHMS}

Most studies demonstrate that transfusion algorithms reduce the need for platelets, fresh-frozen plasma (FFP), or cryoprecipitate. However, any test that prevents empirical administration likely will reduce this need. ${ }^{4}$ Most algorithms suggest transfusion when bleeding is accompanied

From the Department of Anesthesiology, Emory University School of Medicine, Cardiothoracic Anesthesiology and Critical Care, Emory Healthcare, Atlanta, Ga. Disclosures: Authors have nothing to diclose with regard to commercial support.

Received for publication May 13, 2010; revisions received March 6, 2011; accepted for publication March 21, 2011; available ahead of print May 6, 2011.

Address for reprints: Jerrold H. Levy, MD, FAHA, Department of Anesthesiology, Emory University Hospital, 1364 Clifton Rd, NE, Atlanta, GA 30322 (E-mail: Jlevy01@emory.edu).

0022-5223/\$36.00

Copyright (c) 2011 by The American Association for Thoracic Surgery doi:10.1016/j.jtcvs.2011.03.015 by a prothrombin time/activated partial thromboplastin time more than 1.5 times normal, a platelet count less than 50 to 100,000 , or fibrinogen concentration less than $100 \mathrm{mg} / \mathrm{dL}(1 \mathrm{~g} / \mathrm{L}) .^{5}$ Measurement of D-dimers or fibrin degradation products is also frequently done to see whether fibrinolysis is occurring. Because most laboratory testing is slow, point of care testing has been the focus of research as recently reviewed. ${ }^{5}$ Thromboelastography and rotational thromboelastometry are useful to determine fibrinolysis, and thromboelastography or rotational thromboelastometrybased algorithms have been shown to decrease blood product use. However, the machines require quality-control followup and are not available everywhere. Platelet function testing has also been reported but is problematic because most tests need a relatively high platelet count to work and may not be applicable to post-cardiopulmonary bypass (CPB) platelet dysfunction.

\section{TRANSFUSION THERAPY FOR BLEEDING}

Volume replacement after cardiac surgery with critical bleeding may require large fluid volumes. RBC transfusions provide oxygen-carrying capacity, but crystalloids, colloids, and RBCs provide neither coagulation factors nor platelets and can exacerbate coagulopathy by dilution. Thrombocytopenia often follows volume replacement, and the inability to evaluate platelet function complicates matters. Severe bleeding requires FFP, platelets, cryoprecipitate, and potentially factor concentrates (eg, fibrinogen and prothrombin complex concentrates [PCCs]) to restore circulating levels of hemostasis factors. After massive transfusion therapy, hypothermia and acidosis frequently occur, further complicating bleeding. Blood temperature and $\mathrm{pH}$ must be monitored and corrected during any ongoing transfusion effort. Perioperative blood conservation guidelines in cardiac surgery have been published, recommending institution-specific 

Abbreviations and Acronyms
$\mathrm{CPB}=$ cardiopulmonary bypass
FFP $=$ fresh-frozen plasma
FXIII $=$ Factor XIII
PCC = prothrombin complex concentrate
$\mathrm{RBC}=$ red blood cell
rFVIIa $=$ recombinant activated factor VIIa
TXA $=$ tranexamic acid

transfusion algorithms, point-of-care testing, and a multimodal approach to coagulopathy treatment. ${ }^{6}$

Massive transfusion coagulopathy, generally defined as 10 RBC units or more transfused in a 24-hour period, is best studied from trauma literature. Because several blood volumes may be replaced in these patients by the time results are available, laboratory testing is problematic. As a result, transfusion protocols have been developed where fixed doses of FFP and platelets are administered after a specific number of RBC units have been given, often in a 1:1:1 ratio. ${ }^{4}$ Whether these fixed ratios prevent coagulopathy is not established in cardiac surgery, but in trauma and in noncardiac surgical battlefield conditions, fixed transfusion ratios improve survival. ${ }^{7,8}$ Thus, with life-threatening hemorrhage, transfusion of fixed ratios of RBCs, FFP, and platelets should be administered when situations analogous to trauma situations are encountered, such as coagulopathy after complex aortic surgery.
ANTIFIBRINOLYTIC AGENTS: LYSINE ANALOGS

The lysine analogs epsilon aminocaproic acid and tranexamic acid (TXA) competitively inhibit activation of plasminogen to plasmin, an enzyme that degrades fibrin clots. TXA also inhibits plasmin at higher doses, and most studies reported in cardiac surgery involve TXA. Epsilon aminocaproic acid does not consistently reduce transfusion requirements or surgical reexploration. ${ }^{9}$ Multiple meta-analyses of randomized controlled trials consistently report a decrease in bleeding with these agents, but data regarding safety are limited. Postoperative convulsive seizures at one institution were reported to increase from $1.3 \%$ to $3.8 \%$ after cardiac surgery, temporally coincident with high-dose TXA. ${ }^{10}$ In 24 patients with postoperative seizures, all received high doses of TXA intraoperatively from 61 to $259 \mathrm{mg} / \mathrm{kg}$, mean ages were 70 years, and 21 of 24 had open-chamber procedures. ${ }^{10}$ Although these agents are typically used during CPB, additional use of them should be considered postoperatively with evidence of ongoing fibrinolysis.

\section{ANTIFIBRINOLYTIC AGENTS: APROTININ}

Aprotinin, a polypeptide serine protease inhibitor, inhibits plasmin and other serine proteases. In cardiac surgery, multiple randomized, placebo-controlled trials reported that aprotinin reduced bleeding and allogeneic transfusions. ${ }^{11}$ However, recent reports from observational databases and one randomized study questioned the safety of aprotinin. ${ }^{12}$

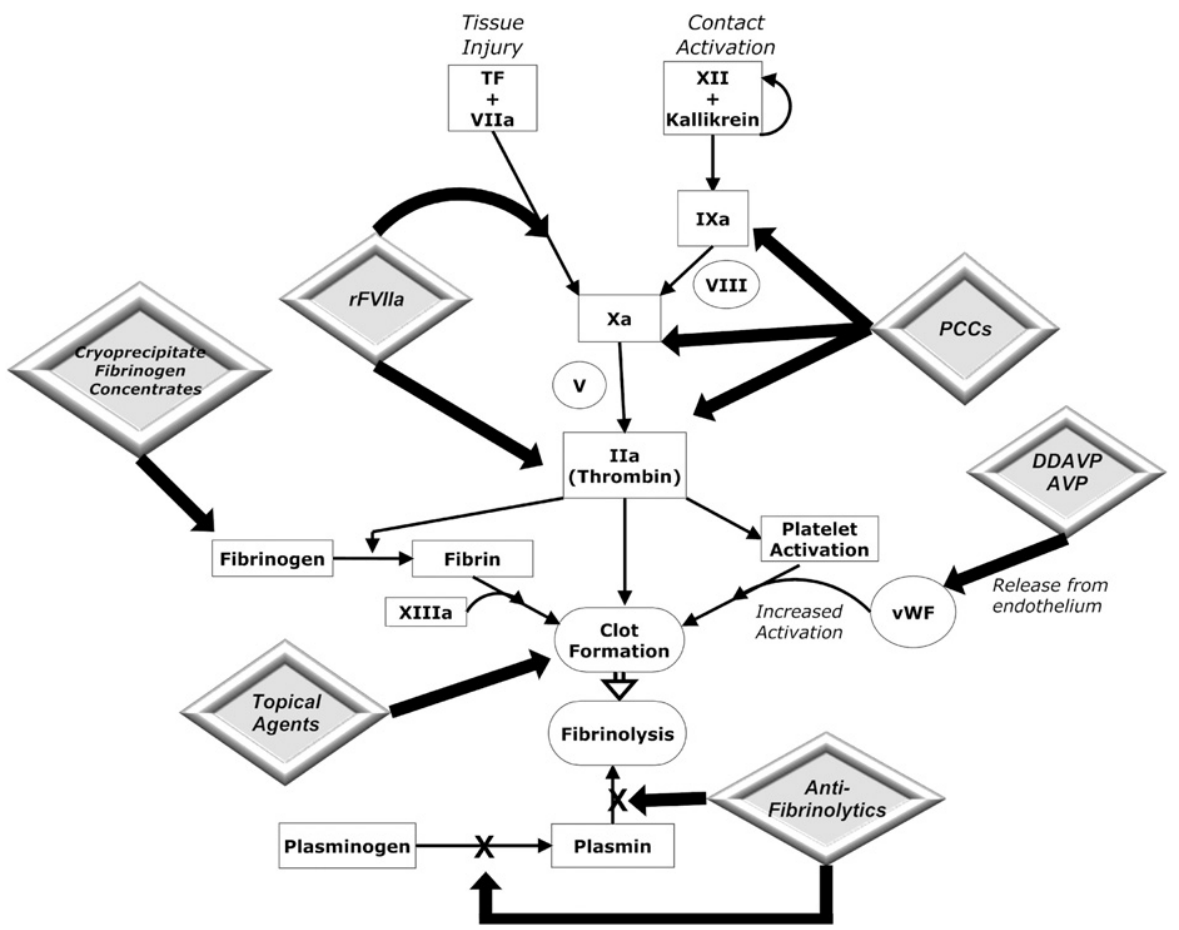

FIGURE 1. An overview of clot formation, demonstrating points of intervention. $T F$, Tissue factor; $r F V I I a$, recombinant factor VII; $P C C$, prothrombin complex concentrates; $A V P$, arginine vasopressin; $v W F$, von Willebrand factor. 
After publication of the BART study, ${ }^{13}$ Bayer Pharmaceuticals (Montville, NJ) removed the drug from marketing, although it is still available for compassionate use. Karkouti and colleagues ${ }^{14}$ reported a retrospective single-center cohort study of 15,365 cardiac surgical patients, of whom 1017 received aprotinin and 14,358 received TXA. They noted aprotinin had a better risk-benefit profile than TXA in high-risk, but not low- to moderate-risk, patients and suggested its use in high-risk cases may be warranted. Other synthetic serine protease inhibitors, such as CU-2010, which possess antifibrinolytic activity comparable to aprotinin, are currently under development. ${ }^{15}$

\section{PROTAMINE}

Protamine is a polypeptide that contains approximately $70 \%$ arginine residues and inactivates the acidic unfractionated heparin, but not low-molecular-weight heparin, molecule via a simple acid-base interaction. ${ }^{16}$ Most patients receive too much protamine for anticoagulation reversal because fixed-dose regimens are based on initial or total heparin dose and do not account for elimination. Excess protamine should be avoided when reversing heparin because it inhibits platelets and proteases, and potentially contributes to coagulopathy. ${ }^{17}$ Maintaining heparin levels during CPB and administering protamine on the basis of the exact circulating heparin level reduced postoperative bleeding and transfusions and produced the lowest activated clotting time values. ${ }^{18}$

Heparin rebound can occur after initial reversal and occurs 2 to 3 hours later postoperatively. ${ }^{19}$ Heparin levels with rebound may range from 0.1 to $0.3 \mathrm{IU} / \mathrm{mL}$, suggesting circulating levels of heparin, based on a 5-L blood volume, of 500 to 1500 units. Protamine doses of 5 to $15 \mathrm{mg}$ may be effective at reversing heparin rebound rather than the $50 \mathrm{mg}$ commonly administered. The activated clotting time is not a sensitive indicator of heparin rebound because platelet counts and fibrinogen levels may also affect values.

Protamine can cause adverse reactions, including anaphylaxis, acute pulmonary vasoconstriction, right ventricular failure, and hypotension. ${ }^{16}$ Patients at an increased risk for adverse reactions are sensitized, often from neutral-protamineHagedorn insulin. Protamine reactions occur in $0.6 \%$ to $2 \%$ of neutral-protamine-Hagedorn insulin-dependent diabetic patients. ${ }^{20,21}$ Other individuals reported at risk for protamine reactions include patients with vasectomy, multiple drug allergies, and prior protamine exposure. ${ }^{16}$

\section{DESMOPRESSIN}

Desmopressin (DDAVP; Sanofi-Aventis, Bridgewater, $\mathrm{NJ}$ ) is the V2 analog of arginine vasopressin that stimulates von Willebrand factor multimers released from endothelial cells. von Willebrand factor mediates platelet adherence to vascular subendothelium by functioning as a protein bridge between glycoprotein Ib receptors on platelets and subendo- thelial vascular basement membrane proteins. Desmopressin (DDAVP) shortens the bleeding time of patients with mild forms of hemophilia A or von Willebrand's disease. Desmopressin (DDAVP) is administered intravenously at a dose of $0.3 \mathrm{mg} / \mathrm{kg}$ over 15 to 30 minutes to avoid hypotension. ${ }^{22}$

Most studies have not confirmed the early reported efficacy during complex cardiac surgery, and a recent review showed only a small effect on perioperative blood loss (median decrease, $115 \mathrm{~mL}$ ). ${ }^{23}$ Because critically ill patients are often receiving vasopressin, which also has V2- and V1mediated effects, there is little benefit to adding desmopres$\sin (\mathrm{DDAVP})$.

\section{FIBRINOGEN}

Fibrinogen is an under-recognized coagulation factor critical for producing effective clot in surgical patients, and data support it as a predictor of perioperative bleeding. ${ }^{24}$ Normal fibrinogen levels are 200 to $400 \mathrm{mg} / \mathrm{dL}$, although the target fibrinogen level in a bleeding patient is not known. Bleeding increases for each $100 \mathrm{mg} / \mathrm{dL}$ decrease in fibrinogen level in parturient patients, who normally have levels more than 400 $\mathrm{mg} / \mathrm{dL},{ }^{25}$ and low fibrinogen levels can predict bleeding after prolonged $\mathrm{CPB} .{ }^{26}$

Most transfusion algorithms recommend therapy when fibrinogen levels are less than $100 \mathrm{mg} / \mathrm{dL}$; however, low fibrinogen levels can increase prothrombin time/partial thromboplastin time that may not correct with FFP. In this situation, cryoprecipitate or fibrinogen concentrates are a better option to restore adequate plasma levels ( $\sim 200 \mathrm{mg} / \mathrm{dL}$ ) because they cause less hemodilution. Fibrinogen can be repleted by cryoprecipitate; 1 unit/10 kg increases fibrinogen by 50 to $70 \mathrm{mg} / \mathrm{dL}$. In Europe, fibrinogen concentrates are available and cryoprecipitate is not used. A fibrinogen concentrate (RiaSTAP; CSL Behring, King of Prussia, $\mathrm{Pa}$ ) has just been granted licensing as an orphan drug for treating bleeding in patients with congenital afibrinogenemia or hypofibrinogenemia.

\section{RECOMBINANT COAGULATION PRODUCTS}

Recombinant proteins are increasingly available for managing bleeding, topical hemostasis, and other therapeutic interventions. Recombinant proteins also can be modified to alter specific characteristics that may be important in therapeutic effects or provide quantities that can be administered supraphysiologically as a therapeutic agent. Currently, they are used to manage bleeding in patients with hemophilia, von Willebrand's disease, or acquired antibodies/inhibitors.

\section{Recombinant Activated Factor VIIa}

Recombinant activated factor VIIa (rFVIIa; NovoSeven, Novo Nordisk, Bagsværd, Denmark) is approved for 
TABLE 1. Postoperative rescue therapy with off-label use of recombinant factor VII in patients undergoing cardiac surgery ${ }^{30,31}$

- Severe $(1 \mathrm{~L} / \mathrm{h})$ or life-threatening bleeding without surgical source of bleeding

- Marginal response to routine hemostatic therapy (ie, platelets, FFP, cryoprecipitate, desmopressin [DDAVP; Sanofi-Aventis, Bridgewater, $\mathrm{NJ}]$ )

- Administration with appropriate hemostatic factors present

- Consider lower doses than used in hemophilia (30-40 $\mu \mathrm{g} / \mathrm{kg})$

- Use in patients with multiple antibodies to platelets; cross-match issues when factors or platelets are not available

- Potential use for Jehovah's Witnesses

patients with hemophilia with inhibitors to treat bleeding but is increasingly used off-label as a prohemostatic agent for life-threatening hemorrhage. ${ }^{27}$ Only approximately $1 \%$ of circulating FVII is activated, and it has no effect until bound with tissue factor. The prohemostatic effects of rFVIIa are produced in part by complexing with tissue factor expressed at the vascular injury site to locally produce thrombin and amplify hemostatic activation. Multiple publications report off-label rFVIIa use after cardiac surgery, although the therapeutic rFVIIa dose in patients without hemophilia has not been established.

Thromboembolic complications with rVIIa use have been reported to be $11.1 \%{ }^{28}$ However, the drug is typically administered as rescue therapy to patients who have already received multiple transfusions, have impaired coagulation, and are at high risk for adverse events. Only arterial thromboembolic event rates were higher after rFVIIa versus placebo (5.5\% vs $3.2 \%$ ) compared with venous thromboembolic events $(5.3 \%$ vs $5.7 \%)$, and were higher among the elderly, likely reflecting the studies in intracranial hemorrhage. ${ }^{28}$

In a recent cardiac surgical study, patients bleeding more than $200 \mathrm{~mL} / \mathrm{h}$ postoperatively were randomized to placebo $(\mathrm{n}=68), 40 \mu \mathrm{g} / \mathrm{kg}$ rFVIIa $(\mathrm{n}=35)$, or $80 \mu \mathrm{g} / \mathrm{kg}$ rFVIIa $(n=69) .{ }^{29}$ The primary end points were critical serious adverse events. Secondary end points included reoperation rates, blood loss, and transfusions. Although more adverse events occurred in the rFVIIa groups, they did not reach statistical significance (placebo, 7\%; 40 $\mu \mathrm{g} / \mathrm{kg}, 14 \% ; P=.25 ; 80 \mu \mathrm{g} / \mathrm{kg}, 12 \% ; P=.43)$. However, after randomization, significantly fewer patients in the rFVIIa group underwent a reoperation because of bleeding $(P=.03)$ or required allogeneic transfusions $(P=.01) .{ }^{29}$ Guidelines for off-label use of rVIIa are given in Table $1 .^{30,31} \mathrm{~A}$ clinical protocol for bleeding incorporating the above concepts is presented in Figure 2.

\section{Factor XIII}

Plasma factor XIII (FXIII) is an important final step in clot formation that stabilizes the initial clot. Several investigators have demonstrated reductions in FXIII during $\mathrm{CPB}$ and an inverse relationship between postoperative

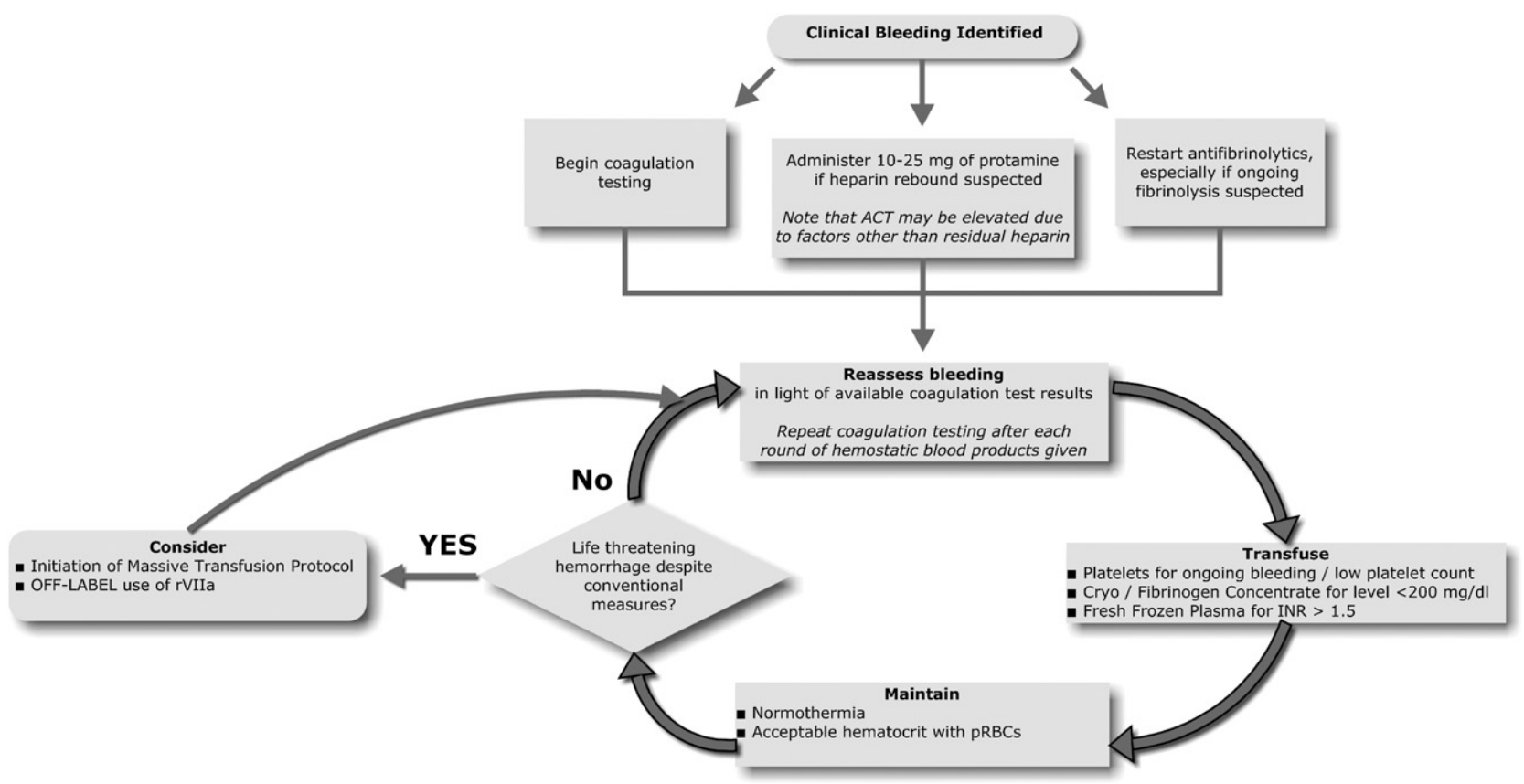

FIGURE 2. Management steps for treatment of excessive postoperative bleeding without an identifiable surgical cause. Note that there is a continual cycle of transfusion and reassessment of bleeding severity, and the decision to transfuse should not be based on laboratory values alone. Consideration of fixed ratio protocols and off-label use of recombinant factor VIIa also needs to be done when conventional measures fail. ACT, Activated clotting time; Cryo, cryoprecipitate; $p R B C s$, packed red blood cells; $r F V I I a$, recombinant factor VII. 
bleeding and FXIII levels. Plasma-derived FXIII (Fibrogammin; CSL Behring, Marburg, Germany) at the end of CPB with concurrent antifibrinolytic therapy reduced postoperative hemorrhage and transfusion requirement in 2 trials $^{32,33}$ including 22 and 75 patients, respectively. The addition of 2500 U FXIII (Fibrogammin) quickly restored the plasma level of FXIII, as measured by Berichrom, to 70 or greater, and reduced transfusion requirements. A recombinant FXIII has been recently reported in clinical studies, and more studies are under way to evaluate this factor as a therapy to reduce bleeding. ${ }^{34}$

\section{Prothrombin Complex Concentrates}

PCCs are concentrates of coagulation factors that include factors II, VII, IX, and $\mathrm{X}$ in variable concentrations. ${ }^{35}$ Two agents (eg, Beriplex P/N [CSL Behring] and Octaplex [Octapharma, Vienna, Austria]) are approved outside the United States for vitamin K antagonist-induced (ie, warfarin) reversal and are being studied currently in the United States. The PCCs available in the United States include FEIBA VH (Baxter, Deerfield, Ill), Profilnine SD (Grifols, Barcelona, Spain), and Bebulin VH (Baxter). They are approved for prevention and control of bleeding in patients with hemophilia B and contain mainly factor IX ${ }^{35}$ Only FEIBA contains factor VII in an activated form, and Bebulin contains only low levels of factor VII. In general, it is considered preferable to give a PCC containing all 4 vitamin $\mathrm{K}$-dependent coagulation factors and natural anticoagulants antithrombin and activated protein $\mathrm{C}$ for anticoagulation reversal. Compared with FFP, PCCs provide quicker international normalized ratio correction, have a small infusion volume, and do not require crossmatching. Although there are historical concerns about potential thrombotic risk with PCCs, present-day PCCs are much improved.

\section{TOPICAL HEMOSTATIC AGENTS}

Topical hemostatic agents are used intraoperatively to promote hemostasis at the vascular injury site. They are classified on the basis of their mechanism of action and include physical or mechanical agents, caustic agents, biologic physical agents, and physiologic agents. ${ }^{36}$ The agent to use depends on the type of bleeding, the agent's specific mechanism of action, its interaction with the environment, and the underlying coagulopathy. ${ }^{36}$ Absorbable agents include gelatin sponges (Gelfoam; Pfizer, New York, NY), derived from purified pork skin gelatin that increase contact activation to help create topical clot. Surgicel (Ethicon, Somerville, NJ) or Oxycel (Becton Dickinson Infusion Therapy Systems, Sandy, Utah) is oxidized regenerated cellulose that works like Gelfoam. Avitene (Davol, Warwick, $\mathrm{RI}$ ) is microfibrillar collagen derived from bovine skin. Collagen sponges are available in different commercial forms and are derived from bovine Achilles tendon or bovine skin. Gelatin foam should not be used near nerves or in confined spaces but can be administered topically with thrombin. CoSeal (Baxter) is used where swelling and expansion are not a concern. BioGlue (Bioform Medical, San Mateo, Calif) has been used in cardiac surgery, but it contains a glutaraldehyde component that cross-links proteins and fixes the tissues to which it is applied.

Topically applied thrombin preparations are also used extensively. The first available thrombin was derived from bovine plasma (Thrombin-JMI, King Pharmaceuticals, Bristol, Tenn). Bovine thrombin currently should be avoided because of its potential for antibovine thrombin antibody formation and immune-mediated coagulopathy. ${ }^{37}$ Currently, there are 2 human thrombins available for clinical use, including plasma-derived thrombin (Evithrom; Omrix, Somerville, NJ, and Johnson \& Johnson, New Brunswick, NJ) and recombinant human thrombin RECOTHROM (ZymoGenetics, Inc, Seattle, Wash).

Fibrin sealants, also referred to as biological glue or fibrin tissue adhesives, are component products that combine thrombin (mostly human) and fibrinogen (usually plasma derived). The first commercial fibrin sealant, Tisseel (Baxter), was approved in 1989. Additional fibrin sealants are currently in use, including Crosseal (Ethicon), Evicel (Ethicon), and FloSeal (Baxter). They are packaged with a dual-syringe delivery system that combines the components to form a fibrin clot. ${ }^{36}$ The thrombin concentration determines the onset and the tensile strength of the fibrin seal. ${ }^{36}$ Crosseal contains human fibrinogen, human thrombin, and TXA. Evicel does not contain any fibrinolytic inhibitors. Agents studied in cardiac surgery, including FloSeal, are the subject of a recent review. ${ }^{38}$

\section{CONCLUSIONS}

The potential for bleeding in patients undergoing cardiac surgery represents an ongoing problem for clinicians. The increasing use of anticoagulation agents creates a need for multiple pharmacologic approaches. The growing use of clopidogrel, prasugrel, and newer oral anticoagulants will continue to pose new paradigms and potential problems in managing surgical patients. Newer therapies, including recombinant therapies, provide clinicians with the ability to administer key coagulation proteins to treat hemorrhage when standard therapies are ineffective.

\footnotetext{
References

1. Hein OV, Birnbaum J, Wernecke KD, Konertz W, Jain U, Spies C. Three-year survival after four major post-cardiac operative complications. Crit Care Med. 2006;34:2729-37.

2. Moulton MJ, Creswell LL, Mackey ME, Cox JL, Rosenbloom M. Reexploration for bleeding is a risk factor for adverse outcomes after cardiac operations. J Thorac Cardiovasc Surg. 1996;111:1037-46.

3. Christensen MC, Krapf S, Kempel A, von Heymann C. Costs of excessive postoperative hemorrhage in cardiac surgery. J Thorac Cardiovasc Surg. 2009;138: 687-93.
} 
4. Stainsby D, MacLennan S, Thomas D, Isaac J, Hamilton PJ. Guidelines on the management of massive blood loss. Br J Haematol. 2006;135:634-41.

5. Steiner ME, Despotis GJ. Transfusion algorithms and how they apply to blood conservation: the high-risk cardiac surgical patient. Hematol Oncol Clin North Am. 2007;21:177-84.

6. Ferraris VA, Ferraris SP, Saha SP, et al. Perioperative blood transfusion and blood conservation in cardiac surgery: the Society of Thoracic Surgeons and The Society of Cardiovascular Anesthesiologists clinical practice guideline. Ann Thorac Surg. 2007;83:S27-86.

7. Dente CJ, Shaz BH, Nicholas JM, et al. Improvements in early mortality and coagulopathy are sustained better in patients with blunt trauma after institution of a massive transfusion protocol in a civilian level I trauma center. I Trauma. 2009;66:1616-24

8. Holcomb JB, Wade CE, Michalek JE, et al. Increased plasma and platelet to red blood cell ratios improves outcome in 466 massively transfused civilian trauma patients. Ann Surg. 2008;248:447-58.

9. Levi M, Cromheecke ME, de Jonge E, et al. Pharmacological strategies to decrease excessive blood loss in cardiac surgery: a meta-analysis of clinically relevant endpoints. Lancet. 1999;354:1940-7.

10. Martin K, Wiesner G, Breuer T, Lange R, Tassani P. The risks of aprotinin and tranexamic acid in cardiac surgery: a one-year follow-up of 1188 consecutive patients. Anesth Analg. 2008;107:1783-90.

11. Sedrakyan A, Wu A, Sedrakyan G, Diener-West M, Tranquilli M, Elefteriades J. Aprotinin use in thoracic aortic surgery: safety and outcomes. J Thorac Cardiovasc Surg. 2006;132:909-17.

12. Mangano DT, Tudor IC, Dietzel C. The risk associated with aprotinin in cardiac surgery. N Engl J Med. 2006;354:353-65.

13. Fergusson DA, Hebert PC, Mazer CD, et al. A comparison of aprotinin and lysine analogues in high-risk cardiac surgery. N Engl J Med. 2008;358:2319-31.

14. Karkouti K, Wijeysundera DN, Yau TM, McCluskey SA, Tait G, Beattie WS. The risk-benefit profile of aprotinin versus tranexamic acid in cardiac surgery. Anesth Analg. 2010;110:21-9.

15. Szabo G, Veres G, Radovits T, et al. The novel synthetic serine protease inhibitor CU-2010 dose-dependently reduces postoperative blood loss and improves postischemic recovery after cardiac surgery in a canine model. $J$ Thorac Cardiovasc Surg. 2010;139:732-40.

16. Levy JH, Adkinson NF Jr. Anaphylaxis during cardiac surgery: implications for clinicians. Anesth Analg. 2008;106:392-403.

17. Mochizuki T, Olson PJ, Szlam F, Ramsay JG, Levy JH. Protamine reversal of heparin affects platelet aggregation and activated clotting time after cardiopulmonary bypass. Anesth Analg. 1998;87:781-5.

18. Despotis GJ, Joist JH, Hogue CW Jr, et al. The impact of heparin concentration and activated clotting time monitoring on blood conservation. A prospective, randomized evaluation in patients undergoing cardiac operation. J Thorac Cardiovasc Surg. 1995;110:46-54.

19. Kuitunen AH, Salmenpera MT, Heinonen J, Rasi VP, Myllyla G. Heparin rebound: a comparative study of protamine chloride and protamine sulfate in patients undergoing coronary artery bypass surgery. J Cardiothorac Vasc Anesth. 1991;5:221-6.
20. Levy JH, Zaidan JR, Faraj B. Prospective evaluation of risk of protamine reactions in patients with NPH insulin-dependent diabetes. Anesth Analg. 1986;65: 739-42.

21. Levy JH, Schwieger IM, Zaidan JR, Faraj BA, Weintraub WS. Evaluation of patients at risk for protamine reactions. J Thorac Cardiovasc Surg. 1989;98:200-4.

22. de Prost D, Barbier-Boehm G, Hazebroucq J, et al. Desmopressin has no beneficial effect on excessive postoperative bleeding or blood product requirements associated with cardiopulmonary bypass. Thromb Haemost. 1992;68:106-10.

23. Mannucci PM, Levi M. Prevention and treatment of major blood loss. $N$ Engl J Med. 2007;356:2301-11.

24. Blome M, Isgro F, Kiessling AH, et al. Relationship between factor XIII activity, fibrinogen, haemostasis screening tests and postoperative bleeding in cardiopulmonary bypass surgery. Thromb Haemost. 2005;93:1101-7.

25. Charbit B, Mandelbrot L, Samain E, et al. The decrease of fibrinogen is an early predictor of the severity of postpartum hemorrhage. J Thromb Haemost. 2007;5:266-73

26. Karlsson M, Ternstrom L, Hyllner M, Baghaei F, Nilsson S, Jeppsson A. Plasma fibrinogen level, bleeding, and transfusion after on-pump coronary artery bypass grafting surgery: a prospective observational study. Transfusion. 2008;48 2152-8.

27. Steiner ME, Key NS, Levy JH. Activated recombinant factor VII in cardiac surgery. Curr Opin Anaesthesiol. 2005;18:89-92.

28. Levi M, Levy JH, Andersen HF, Truloff D. Safety of recombinant activated factor VII in randomized clinical trials. $N$ Engl J Med. 2010;363:1791-800.

29. Gill R, Herbertson M, Vuylsteke A, et al. Safety and efficacy of recombinant activated factor VII: a randomized placebo-controlled trial in the setting of bleeding after cardiac surgery. Circulation. 2009;120:21-7.

30. Sniecinski RM, Chen EP, Levy JH, Szlam F, Tanaka KA. Coagulopathy after cardiopulmonary bypass in Jehovah's Witness patients: management of two cases using fractionated components and factor VIIa. Anesth Analg. 2007;104 763-5.

31. Despotis G, Avidan M, Lublin DM. Off-label use of recombinant factor VIIA concentrates after cardiac surgery. Ann Thorac Surg. 2005;80:3-5.

32. Godje O, Gallmeier U, Schelian M, Grunewald M, Mair H. Coagulation factor XIII reduces postoperative bleeding after coronary surgery with extracorporeal circulation. Thorac Cardiovasc Surg. 2006;54:26-33.

33. Godje O, Haushofer M, Lamm P, Reichart B. The effect of factor XIII on bleeding in coronary surgery. Thorac Cardiovasc Surg. 1998;46:263-7.

34. Levy JH, Gill R, Nussmeier NA, et al. Repletion of factor XIII following cardiopulmonary bypass using a recombinant A-subunit homodimer. A preliminary report. Thromb Haemost. 2009;102:765-71.

35. Levy JH, Tanaka KA, Dietrich W. Perioperative hemostatic management of patients treated with vitamin K antagonists. Anesthesiology. 2008;109:918-26.

36. Achneck HE, Sileshi B, Jamiolkowski RM, Albala DM, Shapiro ML, Lawson JH A comprehensive review of topical hemostatic agents: efficacy and recommendations for use. Ann Surg. 2010;251:217-28.

37. Lawson JH. The clinical use and immunologic impact of thrombin in surgery. Semin Thromb Hemost. 2006;32(Suppl 1):98-110.

38. Barnard J, Millner R. A review of topical hemostatic agents for use in cardiac surgery. Ann Thorac Surg. 2009;88:1377-83. 